\title{
The Fertility Transition Around the World - 1950-2005
}

\author{
Holger Strulik ${ }^{\dagger}$ and Sebastian Vollmer** \\ Leibniz Universität Hannover, Discussion Paper No. 443 \\ ISSN 0949-9962
}

March 2010

\begin{abstract}
In this paper we analyze the distribution of fertility rates across the world using parametric mixture models. We demonstrate the existence of twin peaks and the division of the world's countries in two distinct components: a high-fertility regime and a low fertility regime. Whereas the significance of twin peaks vanishes over time, the two fertility regimes continue to exists over the whole observation period. In 1950 about two thirds of the world's countries belonged to the high-fertility regime and the rest constituted the low-fertility regime. By the year 2005 this picture has reversed. Within both the low- and the high-fertility regime the average fertility rate declined, with a larger absolute decline within the high-fertility regime. Visually, the two peaks moved closer together. For the low fertility-group we find both $\beta$ - and $\sigma$ - convergence but we cannot establish any convergence pattern for the high fertility regime. Overall our findings are difficult to reconcile with the standard view of a fertility trap but they support the "differentiated take-off" view established in the Unified Growth literature.
\end{abstract}

Keywords: Fertility, Convergence, Twin Peaks, Fertility Regimes, Unified Growth.

\footnotetext{
${ }^{*}$ We would like to thank David Bloom and Oded Galor for useful comments and Florian Ketterer for providing the EM-algorithm.

${ }^{\dagger}$ Department of Economics, Brown University, Providence, RI 02912, USA, email: holger_strulik@brown.edu and University of Hannover, Wirtschaftswissenschaftliche Fakultaet, 30167 Hannover, Germany; email: strulik@vwl.uni-hannover.de.

${ }^{* *}$ Harvard University, Center for Population and Development Studies, 9 Bow Street, Cambridge, MA 02138; email: svollmver@hsph.harvard.edu.
} 


\section{INTRODUCTION}

Over the last two hundred years of human history every successfully developing country experienced a fertility transition: starting at initially high levels, fertility rates went down towards a low plateau, sometimes below replacement level. This one-time demographic event seems to be so inevitably linked with economic growth that many researchers are starting to understand the fertility transition as a prerequisite for successful development (see Galor, 2005, for an overview). In almost all cases the fertility transition was lead by a secular fall of mortality rates so that it seems that decreasing mortality rates have caused fertility to fall. However, there are certainly other forces at work as well since the specific pattern of the fertility transition differs substantially across countries (see e.g. Chesnais, 1992, Lee, 2003, Reher, 2003).

The Western European countries and their Western offshoots (the U.S., Canada) experienced the transition first around the end of the nineteenth century. One salient feature of their transition was the chronological proximity between the onsets of mortality decline and fertility decline (0-10 years). This chronological association became much less visible for countries which experienced the fertility transition later. If the fertility decline took off in the 1980s or later it followed the mortality decline with an average delay of 40-45 years (Reher, 2003). Until today, some countries, notably from Sub-Saharan Africa, show so little tendency for declining fertility that the question arises whether the fertility transition is indeed a world-wide phenomenon or whether there exists a "fertility trap" that hinders some countries to follow the path of the historical leaders. ${ }^{1}$

In the economics literature the catch up process is statistically assessed with tests for so called $\beta$ - and $\sigma$-convergence. These tools have been developed in the context of the take off towards long-run income growth (Barro and Sala-i-Martin, 1992). But they are easily adapted to analyze the fertility transition. Here, $\beta$-convergence applies if countries of initially high fertility experience a stronger decline of fertility than countries of initially low fertility. $\sigma$-convergence occurs if the cross-sectional dispersion, measured by the standard deviation of fertility, for a group of countries declines over time. These concepts are not redundant. While $\beta$ convergence implies a tendency for $\sigma$ convergence, it is not sufficient because countries are also affected by

\footnotetext{
${ }^{1}$ The notion of a fertility trap originates from Malthus (1798). Nelson (1956) is a first modern formulation of the idea as a locally stable equilibrium. See also, among others, Koegel and Prskawetz (2001) and Strulik (2004). With a different notion of glacier-slow development (rather than local stability) the Malthusian trap is also discussed in the unified growth literature, see Galor (2005).
} 
fertility unrelated shocks. In turn, the observation of decreasing dispersion does not necessarily entail $\beta$-convergence. In the economics literature it is also an ongoing debate whether the world income distribution is twin-peaked and wether there is "club convergence", i.e. converging income levels within specific groups of countries but diverging income levels between the these groups or "convergence clubs". 2

Recently a couple of articles addressed the problem of converging or non-converging vital rates across the world. Using histograms and inference from eyeballing, Wilson (2001) found twin peaks of the distribution of world-wide population weighted fertility in the 1950s, which vanished over time until the year 2000. From that he concluded that "we are moving into a world where the distinction between developed and developing countries is of greatly diminished relevance to fertility" and that "the overwhelming trend is for low fertility to become a general feature of poor and rich countries". Using the $\beta$ - and $\sigma$-convergence criterion, Dorius (2008) arrived at a much less optimistic conclusion by observing that countries began only recently to converge towards less differentiated fertility rates. Using a set of inequality measures he actually finds evidence for diverging fertility rates over the last half century. In a related study on mortality, Bloom and Canning (2007) find evidence for a twin-peaked distribution and a "mortality trap". Using a mixture model they are able to identify a high mortality and a low mortality regime and estimate the probability of being in the low-mortality regime to be positively related to initial life-expectancy in 1963.

Here we take up from Bloom and Canning (2007) the idea of the world being divided in different regimes and apply it to the fertility transition. Using a parametric mixture model we are able to scrutinize these earlier convergence results. The method is particularly useful since it does not a priori assign the world's countries into different groups by imposing a certain threshold, nor does it impose a particular assumption on the number of "convergence clubs".

Our results provide a rejoinder of the previous conflicting views on the fertility transition and some interesting further results. Using modern econometric methods we confirm that the world's fertility distribution became indeed single-peaked after 1990. At the same time, however, we also firmly established that from the beginning of our observation period in 1950 until the end in 2005

\footnotetext{
${ }^{2}$ The twin peak debate originated from Quah (1993, 1996), see also Jones (1997), Kremer et al. (2001), and Feyrer (2008). For a comprehensive introduction of $\beta$ and $\sigma$-convergence see Chapter 11 of Barro and Sala-iMartin (2004), for a broader discussion of convergence and convergence clubs see also Baumol (1986), Durlauf and Johnson (1995), Azariades (1996), Galor (1996), Pritchett (1997), and Pomeranz (2000). For the debate on a low level equilibrium or poverty trap see, among many others, Bloom and Canning (2003), Graham and Temple (2006), and Kray and Radatz (2007).
} 
there exist two distinct components of the world fertility distribution: a high-fertility regime and a low-fertility regime. Within both regimes fertility is falling over time albeit starting from a much higher initial level in the high fertility regime. We also observe $\sigma$-convergence across the world and within the low fertility regime but not within the high-fertility regime. Furthermore we show $\beta$-convergence within the low-fertility regime but not within the high fertility regime.

These findings suggest the following assessment of the world fertility transition. For countries within the low fertility regime there exists a strong tendency to converge towards a common low fertility rate below replacement level. Initial fertility is a good predictor for future fertility decline. The high-fertility regime, on the other hand, is not a convergence club and, consequently, it is difficult to conceptualize the countries belonging to this regime as being stuck in a "highfertility" trap or, more formally, approaching a locally stable high-fertility-equilibrium. This view is substantiated by the fact that most countries in the high-fertility group also experience some decrease of fertility over time and, more importantly, by the fact that between 1950 and 2005 altogether 49 high-fertility countries were able to enter the low-fertility regime. Moreover, initial fertility is not a good predictor for leaving the high-fertility group.

This assessment of the world fertility transition supports recent insights from unified growth theory (see Galor 2005, 2009 for overviews). Using dynamic general equilibrium models with demographic-economic feedback effects this literature argues that the view of the world as being divided into clubs of countries diverging toward different locally stable equilibria, with initial conditions (initial fertility rates) determining the direction of development, is misleading. Instead, unified growth theory suggests that all countries evolved from an epoch of quasi-stagnation and high fertility towards high growth and low fertility and that geographic and biological fundamentals determine the timing of the take off towards high growth and low fertility. ${ }^{3}$

In other words, unified growth theory conceptualizes the world as divided in different regimes, within one regime the take off is not yet visible, within the other regime we see convergence. Potentially there could be a third regime consisting of countries on the way from the highfertility regime towards the low-fertility regime. Given that the movement between low and high-fertility regime is sufficiently fast and/or that at each time interval there are sufficiently few countries "on the move", the movers are not discernable as a separate group, and there

\footnotetext{
${ }^{3}$ The unified growth view originates from Galor and Weil (2000). The differentiated take-off view was popularized by Lucas (2000). See Strulik (2008a,b) for a theoretical approach on the geographical distribution of the onset of fertility transition.
} 
are just two regimes. In contrast to the fertility trap view, however, a country's association with the not-yet-converging regime is potentially temporary. A country's initial fertility rate is not a good predictor of the take-off because the differentiated take-off over time originates from fundamentals rather than initial values. Only for countries which successfully entered the low-fertility regime the theory predicts convergence as fertility rates during the initiated fertility transition approach a low-level. This is exactly what we find confirmed in the data.

\section{DAtA AND Method}

In the economics literature the notion of twin peaks in the cross-country income distribution was introduced by Quah (1996). He interpreted the emergence of twin peaks as polarization of the cross-country income distribution into a rich and a poor convergence club. Diagrammatically, twin peaks can be observed by non-parametric kernel density estimation. But this method leaves open the question of their econometric significance. For that purpose, Silverman (1981) showed that the number of peaks of a kernel density estimator is a right-continuous, monotonically decreasing function of the bandwidth for normal kernels. This allowed him to define the $k$ critical bandwidth as the minimal bandwidth such that the density still has $k$ peaks and not yet $k+1$ peaks. Based on the notion of the $k$-critical bandwidth, Silverman (1981) proposed a bootstrap test for the hypothesis of $k$ peaks against the alternative of more than $k$ peaks. Bianchi (1997) was the first to apply Silverman's test to cross-country income data and he confirmed Quah's hypothesis.

More recently, Holzmann et al. (2007) pointed out that it is misleading to look at the number of peaks of the cross-country income distribution. They show that simple rescaling of the data (e.g. taking logs) produces a statistically significant triple peaked cross-country income distribution. Countries which were previously assigned to Quah's poor convergence club are now considered middle-income on the log-scale, which obviously doesn't make much sense for economic interpretation. Holzmann et al. (2007) propose an alternative methodology which is invariant to strictly monotonic transformation of the data and is thus robust towards this shortcoming of the twin peaks approach. We are going to adopt their approach for the crosscountry distribution of fertility rates.

We use the United Nations World Population Prospects (2008 Revision) to obtain data on the total fertility rate of 184 countries over the period of 1950 to 2005. The data comes in 
eleven intervals of five year length and includes no missing values. Exploratory data analysis with kernel density estimators reveals a twin peak phenomenon similar to income data and thus requires similar techniques to model it.

Following Holzmann et al. (2007) and Vollmer et al. (2009) we model the cross-country distribution of fertility rates as a finite mixture. In a two-component normal mixture, the observations have density

$$
f\left(x ; \alpha, \mu_{1}, \mu_{2}, \sigma_{1}, \sigma_{2}\right)=(1-\alpha) \phi\left(x ; \mu_{1}, \sigma_{1}\right)+\alpha \phi\left(x ; \mu_{2}, \sigma_{2}\right),
$$

with $0 \leq \alpha \leq 1$ and

$$
\phi(x ; \mu, \sigma)=\frac{1}{\sqrt{2 \pi \sigma^{2}}} \exp \left(-\frac{(x-\mu)^{2}}{2 \sigma^{2}}\right) .
$$

We assume without loss of generality that $\mu_{1} \leq \mu_{2} . \phi\left(x ; \mu_{1}, \sigma_{1}\right)$ and $\phi\left(x ; \mu_{2}, \sigma_{2}\right)$ correspond to the distributions of the two assumed sub-populations, and $\alpha$ and $1-\alpha$ are interpreted as their relative sizes.

Note that it is essential to set up a joint model for the two sub-populations, since we want to investigate convergence within the complete distribution. The parameters $\alpha, \mu_{1}, \mu_{2}, \sigma_{1}$ and $\sigma_{2}$ are estimated from the data by the method of maximum likelihood. We allow for unequal variances $\sigma_{1}^{2}$ and $\sigma_{2}^{2}$, because a likelihood ratio test shows that the simplifying assumption of equal variances does not hold for all years.

One of our most important results that we establish below is that a mixture of two one-peaked distributions can have one or two peaks. This is so because two distributions will overlap if they are sufficiently close together such that the peaks of the two underlying distributions will merge into one. Our methodology thus has the additional advantage that it can still identify heterogeneity in cases where multiple peaks are not visible and/or a statistical test for their significance fails. Moreover, we can calculate for each observation the posterior probabilities $p_{1}$ and $p_{2}$ for belonging to the first or respectively second component of the mixture model. This allows us to assign each observation to one of the components depending on which of the two probabilities is higher. Movements between the components are of particular interest, this essentially means that we observe $p_{1}<p_{2}$ in one year and $p_{2}>p_{1}$ in another year.

The likelihood function in finite normal mixtures with different variances is unbounded, thus, a global maximizer of the likelihood function does not exist. However, when using reasonable 
starting values maximization algorithms such as EM or quasi Newton find stable local maxima of the log-likelihood function.

To substantiate our findings it is, of course, essential that we formally validate that there are indeed two components necessary to model the data, i.e. that we test the hypothesis that there are two components against the alternative that a single component is sufficient to describe the world fertility distribution accurately. This turns out to be a quite difficult parametric testing problem; see Chen and Chen (2003) for some history of attempts to solve it. Here, we will use a novel approach, the EM-test developed by Chen and Li (2008) for normal mixtures in mean and variance parameters, a test which overcomes many drawbacks of the simple likelihood ratio test for the same problem. It was first introduced to the economic literature by Vollmer et al. (2009). Details on the methodology are provided by Chen and Li (2008) and Vollmer et al. (2009).

\section{Results}

We begin with testing for the number of components and the number of peaks of the world fertility distribution. The test for the number of components finds that the hypothesis of just one component can be rejected with a $p$-value smaller than 0.01 in all subperiods from 1950 to 2005. The test for the number of peaks is reported in Table 1. Over the whole observation period the hypothesis of two peaks cannot be rejected in favor of more than two peaks. Thus there are at most two peaks. Until 1990 we can firmly reject the hypothesis that there is just one peak. Afterwards the second peak vanishes over time. In subperiod 1990-1995 the second peak is still weakly significant at a 10 percent level, for the two latest intervals it becomes statistically insignificant.

These observations reconcile the seemingly conflicting views on fertility convergence sketched in the Introduction. A vanishing twin peak is compatible with the view of a world divided into two different fertility regimes, one formed by the high-fertility component, the other by the low-fertility component.

Using the results reported in Table 2 we can explain how the two-component distribution morphed from twin-peaked towards single-peaked. Both component-means declined gradually over time. While the mean of the high-fertility component declined from 6.47 to 4.39, the mean of the low fertility component declined from an already low initial mean of 3.14 to 1.89 
Table 1: Silverman's Test for the Number of Peaks

\begin{tabular}{lll}
\hline \hline Years & $p_{1}$ & $p_{2}$ \\
\hline $1950-1955$ & 0.0005 & 0.6475 \\
$1955-1960$ & 0.0005 & 0.3880 \\
$1960-1965$ & 0.0005 & 0.4465 \\
$1965-1970$ & 0.0005 & 0.5230 \\
$1970-1975$ & 0.0025 & 0.9520 \\
$1975-1980$ & 0.0025 & 0.2730 \\
$1980-1985$ & 0.0005 & 0.3075 \\
$1985-1990$ & 0.0010 & 0.7730 \\
$1990-1995$ & 0.0725 & 0.5745 \\
$1995-2000$ & 0.3745 & 0.4620 \\
$2000-2005$ & 0.4835 & 0.4220 \\
\hline
\end{tabular}

We report the $p$-values for the hypothesis of one peak against more than one peaks $\left(p_{1}\right)$ and the $p$-values for the hypothesis of two peaks against the alternative of more than two peaks $\left(p_{2}\right)$.

in 2000-2005, falling below replacement level in 1985-1990. The fact that the mean of the high-fertility component declined faster implies a narrowing gap between the high- and low fertility component. This phenomenon is visualized in Figure 1 which contrasts the estimated distribution for the initial period 1950-1955 with the one for the final period 2000-2005. While two peaks are still visible in 2000-2005 the valley in between has become substantially less deep, this way causing the smaller peak to be statistically insignificant.

Over time a substantial fraction of countries managed to enter the low-fertility regime. Statistically, this can be seen by noting that at the beginning of the observation period more than two-thirds of the countries are associated with the high-fertility component $(\alpha=0.68$ in Table 2). At the end of the observation period the fraction of countries belonging to the high-fertility component decreased below one half $(\alpha=0.49)$.

The continuous move of countries from the high-fertility regime to the low-fertility regime seemingly suggests convergence of fertility rates across countries. A closer look the results, however, provides a more differentiated picture. We observe declining dispersion within the low fertility regime; $\sigma_{1}$ declined by about a third from 0.79 to 0.55 . Within the high-fertility regime, initial dispersion was about the same as within the low-fertility regime. But then, from 1950 to 2005 the standard deviation within the high-fertility regime $\left(\sigma_{2}\right)$ almost doubled from 0.71 to 1.38. Across all countries the standard deviation $(\sigma)$ first increased until 1980 and then declined to a level somewhat below its starting point. 


\section{Figure 1: Cross-Country Distribution of Fertility Rates: 1950-1955 VS. 2000-2005}
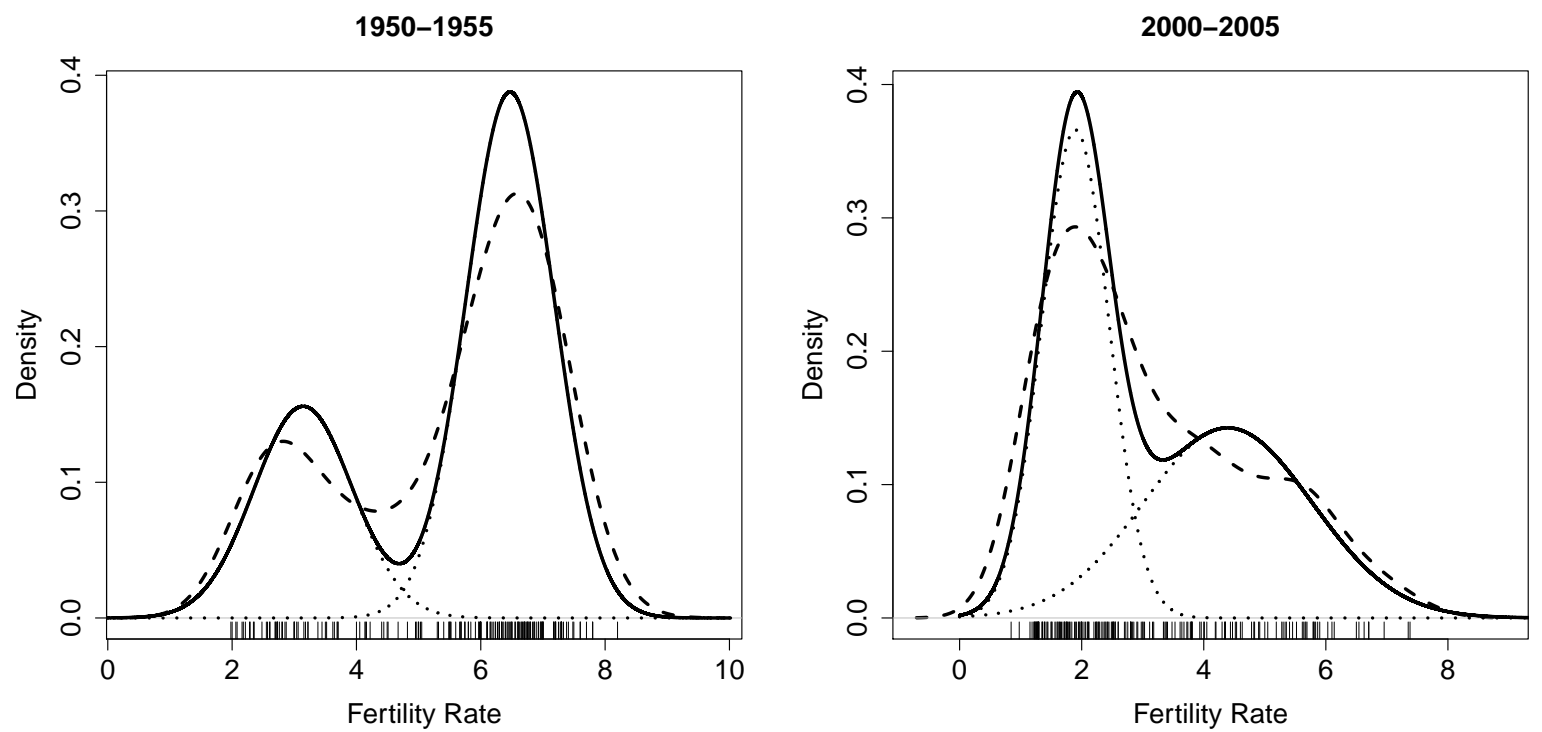

Note: Fitted mixture model (solid line), weighted components (dotted lines), and kernel density estimator (dashed line). The rugs below the plots represent the observed fertility rates.

Table 2: Evolution of the Two Components over Time

\begin{tabular}{llllllll}
\hline \hline Year & $\alpha$ & $\mu_{1}$ & $\mu_{2}$ & $\sigma_{1}$ & $\sigma_{2}$ & $\sigma$ & $p$-value \\
\hline $1950-1955$ & $68.9 \%$ & 3.14 & 6.47 & 0.795 & 0.709 & 1.704 & $<0.01$ \\
$1955-1960$ & $67.4 \%$ & 3.21 & 6.60 & 0.892 & 0.637 & 1.744 & $<0.01$ \\
$1960-1965$ & $69.2 \%$ & 3.02 & 6.57 & 0.801 & 0.704 & 1.795 & $<0.01$ \\
$1965-1970$ & $66.8 \%$ & 2.80 & 6.49 & 0.695 & 0.812 & 1.900 & $<0.01$ \\
$1970-1975$ & $67.4 \%$ & 2.51 & 6.24 & 0.583 & 1.022 & 1.967 & $<0.01$ \\
$1975-1980$ & $68.4 \%$ & 2.18 & 5.85 & 0.467 & 1.287 & 2.023 & $<0.01$ \\
$1980-1985$ & $68.5 \%$ & 2.06 & 5.53 & 0.442 & 1.399 & 2.000 & $<0.01$ \\
$1985-1990$ & $66.9 \%$ & 2.02 & 5.18 & 0.461 & 1.418 & 1.904 & $<0.01$ \\
$1990-1995$ & $69.2 \%$ & 1.83 & 4.63 & 0.397 & 1.485 & 1.803 & $<0.01$ \\
$1995-2000$ & $50.0 \%$ & 2.02 & 4.81 & 0.633 & 1.326 & 1.741 & $<0.01$ \\
$2000-2005$ & $49.4 \%$ & 1.90 & 4.39 & 0.552 & 1.382 & 1.630 & $<0.01$ \\
\hline
\end{tabular}

$\alpha$ is the weight of the high fertility components; $\mu_{1}, \mu_{2}$ are the means and $\sigma_{1}, \sigma_{2}$ are the standard deviations of the two components. $\sigma$ is the standard deviation of the full sample. In the last column we report the $p$-values for the EM of one against two components.

In conclusion, we observe $\sigma$-convergence within the low-fertility regime and (from the 1980s) across all countries but no $\sigma$ convergence within the high fertility regime. This result supports the view of converging fertility behavior within the low-fertility regime as well as world-wide converging fertility behavior, the latter because over time more and more countries manage to enter the low-fertility regime. At the same time we observe diverging fertility behavior within 
the high-fertility regime, a first indication that the high- fertility regime may be difficult to interpret as a locally stable development trap.

The results for 1950-1955 and 2000-2005 are visualized in Figure 1 (c.f. Figure 4 in the appendix for all other years). In 1950-1955 the peaks were equally wide, but the peak of the high fertility regime is much higher than the peak of the low fertility regime because about two thirds of the countries are assigned to the high fertility regime. The overlap of the two distributions is very small. In 2000-2005 the two distributions have approximately the same area $(\alpha \approx 0.5)$. But the peak of the low fertility regime is tall and slim whereas the peak of the high fertility regime is short and wide. The overlap between the two distributions is now substantial. This explains why the second peak is not significant anymore (Table 1) although still discernable in Figure 1.

Figure 2: Cross-Country Distribution of Fertility Rates: 1950-1955 vi. 2000-2005
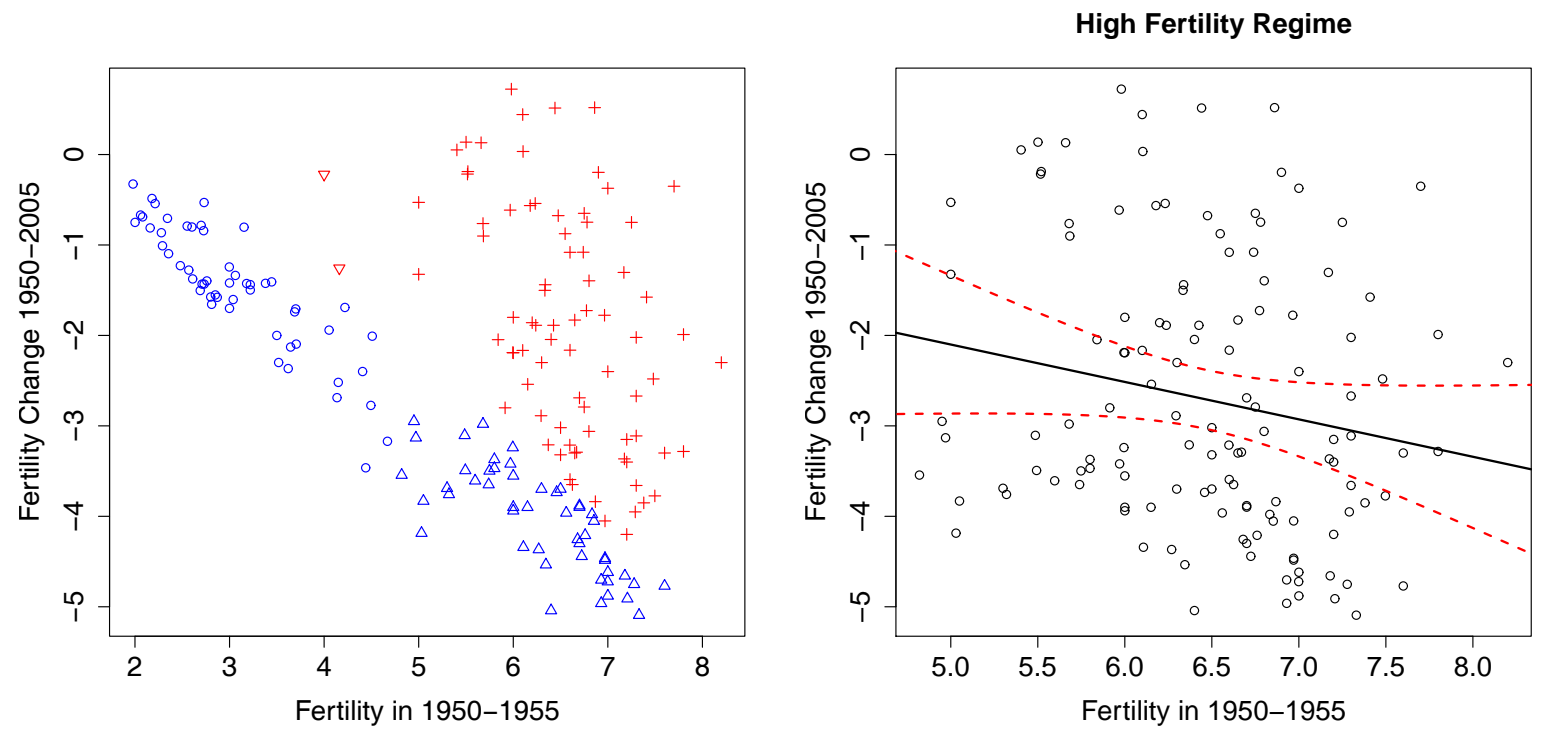

Left hand side: Blue circles: low fertility regime in both periods. Red crosses: high fertility regime in both periods. Triangles: movers between high and low fertility regimes (left figure). Right hand side: $\beta$-convergence regression for the high fertility regime. The dotted red lines represent a $95 \%$ confidence band.

We next investigate whether there is $\beta$-convergence, i.e. conditional $\beta$-convergence of fertility rates within each sample. For that purpose we have regressed the change of fertility rates from 1950 to 2005 on its initial level in 1950-1955 following Barro and Sala-i-Martin (1992). The results are shown in Table 3. 
TABle 3: $\beta$-CONVERGence

\begin{tabular}{llll}
\hline \hline & Full Sample & Low Fertility & High Fertility \\
\hline Intercept & -0.15367 & $0.81592^{* * *}$ & -0.03201 \\
& $(0.31490)$ & $(0.25655)$ & $(1.23266)$ \\
Fertility 50-55 & $-0.39888^{* * *}$ & $-0.72903^{* * *}$ & $-0.41370^{* *}$ \\
& $(0.05516)$ & $(0.08012)$ & $(0.18940)$ \\
\hline
\end{tabular}

Dependent variable: Change of total fertility rate 1950-2005.

For the low fertility regime we find a significantly negative coefficient, which is also economically important: a higher initial fertility rate of one child per woman corresponds an additional 0.73 decline of fertility. This means that, within the low fertility regime, an initial difference of one child per woman is reduced to 0.27 children per woman over the observation period. The coefficient for the high fertility regime is significant as well, but since the standard deviation of the intercept is so high, the result is not meaningful at all. The right hand side panel of Figure 2 substantiates this claim by showing that the 95 percent confidence band around the regression line is very wide for the high fertility regime. In fact the confidence band is so wide that it could engulf a horizontal line or even a line with positive slope. In conclusion, we do not find $\beta$-convergence in the high fertility regime.

We have investigated the movements between the two components in more detail using posterior probabilities. The threshold between the low- and high fertility regime can be obtained endogenously. Graphically, it is the fertility rate where the dotted lines in Figures 1 and 4 intersect. In 1950-1995, only 56 out of 184 countries were assigned to the low fertility regime, in 2000-2005 it is a majority of 103 countries. The results are summarized in the left hand side panel of Figure 2. Blue dots identify the countries that belonged to the low fertility regime in 1950-55 and in 2000-05. The phenomenon of $\beta$ convergence is clearly visible. Note also, that the blue dots can be found quite closely to the estimated regression line over the whole range of fertility rates in the low-fertility regime. Formally, the data supports a linear difference equation for fertility $\Delta F=0.82-0.73 \mathrm{~F}$ with an equilibrium where $\Delta F=0$ and thus at $F=0.82 / 0.73=1.12$. This means that the so far available data suggests the alarming result that the long-run steadystate of the demographic transition is assumed at a fertility rate of 1.12, far below replacement level. A slowing down or reversal of the downward trend at very low fertility rates is not yet visible in the data. 
Blue triangles in the left hand side panel of Figure 2 indicate countries that managed to enter the low-fertility regime between 1950 and 2005. Interestingly, a number of countries with very high initial fertility rates of more than seven managed to move into the low fertility regime whereas some other countries with relatively low fertility rates (compared to the mean within the high-fertility regime), i.e. countries with initial fertility rates around five, remained in the highfertility regime. This lets us conclude that the initial level of fertility is only a good predictor for changes of fertility once the transition towards the low fertility regime has been made. The take off towards the low fertility regime appears to be not predicted by the initial fertility rate. Other, more fundamental forces are at work to determine the chronologically differentiated take off across countries.

In Figure 3 we identify on a world map the countries which stayed in their initial regime and the countries which managed to enter the low-fertility regime. In 1950-1955 most of Latin America (except Argentina), Africa and Asia belonged to the high fertility regime. By 20002005 most of Latin America and East Asia have transitioned into the low fertility regime, Africa (except Morocco, Tunesia, Algeria, and South Africa), the Indian subcontinent and the Arabian peninsula remained in the high fertility regime.

\section{Conclusion}

In this article we have investigated the evolution of the world fertility distribution and scrutinized the important question whether there is convergence of fertility behavior. For that purpose we utilized a recently developed econometric machinery. Our most important findings are that the world was and still is separated into a low-fertility regime and a high-fertility regime and that this finding is consistent with the observation of a vanishing twin-peak. For the low fertility regime we have demonstrated $\beta$ - and $\sigma$-convergence, indicating an ongoing fertility transition and that the countries belonging to this regime are indeed converging towards a common low fertility rate below replacement level.

Within the high-fertility group we find neither $\beta$ convergence nor $\sigma$-convergence. Actually, countries belonging to this group seem to drift farther apart. Thus we find no evidence for a "fertility trap" conceptualized as a locally stable equilibrium of underdevelopment. Again, the notion of an absent fertility trap is consistent with the view of a world separated in different 
fertility regimes. It supports the theoretical literature established by unified growth theory, which argues in favor of a differentiated take off over time (rather than multiple equilibria).

Initial fertility rates are not a good predictor for the successful move towards the low fertility regime. Yet quite a few countries managed this transition over last half century. This raises, of course, the question, which are good predictors for the take off towards the low fertility regime. We leave this question for future research. From inspection of the maps in Figure 3, geographic location seems to be a good predictor, a conclusion that would be consistent with the theory of a geographically differentiated take-off developed in Strulik (2008a,b).

An alarming finding is that our convergence results suggest that the fertility transition within the low fertility regime is still ongoing at unchanged speed, with a predicted equilibrium at 1.12, far below replacement level. Perhaps this assessment is too pessimistic and actual adjustment is non-linear, with undershooting behavior and adjustment towards replacement level from below. This view is theoretically supported by Strulik and Weisdorf (2008). 


\section{REFERENCES}

Azariadis, C., 1996, The economics of poverty traps, part one: Complete markets, Journal of Economic Growth 1, 449-496.

Baumol, W., 1986, Productivity growth, convergence, and welfare, American Economic Review $76,1072-1085$.

Barro, R.J. and X. Sala-i-Martin, Economic Growth, 2nd ed., MIT Press, Cambridge, MA.

Bianchi, M., 1997, Testing for Convergence: Evidence from Non-Parametric Multimodality Tests. Journal of Applied Econometrics 12, 393-409.

Bloom, D.E., D. Canning, and J. Sevilla, 2003, Geography and poverty traps, Journal of Economic Growth 8, 355-378.

Bloom, D.E. and Canning, D., 2007, Mortality traps and the dynamics of health transitions, Proceedings of the National Academy of Sciences 104, 16044-16049.

Chen, H. and J. Chen, 2003, Tests for homogeneity in normal mixtures with presence of a structural parameter, Statistica Sinica 13, 351-365.

Chen, J. and P. Li, 2008, Hypothesis test for Normal Mixture Models: the EM Approach, Annals of Statistics 37, 2523-2542.

Chesnais, J.-C., 1992, The Demographic Transition: Stages, Patterns, and Economic Implications, Oxford: Clarendon Press.

Dorius, S.F., 2008, Global convergence? A reconsideration of changing intercountry inequality in fertility, Population and Development Review 34, 519-537.

Durlauf, S.N. and P. A. Johnson, 1995, Multiple Regimes and Cross-Country Growth Behavior, Journal of Applied Econometrics 10, 365-384.

Galor, O., 1996, Convergence? Inferences from theoretical models, Economic Journal, 10561069.

Galor, O. and D.N. Weil, 2000, Population, Technology, and Growth: From Malthusian Stagnation to the Demographic Transition and Beyond, American Economic Review 90, pp. 806-828. 
Galor, O., 2005, From stagnation to growth: unified growth theory, in: Handbook of Economic Growth, Amsterdam: North-Holland.

Galor, O., 2009, Comparative economic development: insights from unified growth theory, 2008 Lawrence Klein Lecture, Discussion Paper, Brown University.

Graham, B.S. and J.R.W. Temple, 2006, Rich nations, poor nations: how much can multiple equilibria explain?, Journal of Economic Growth 11, 5-41.

Holzmann, H., S. Vollmer and J. Weisbrod, 2007, Twin Peaks or Three Components? Discussion Paper. University of Göttingen. Revised December 2009.

Jones, C.I., 1997, On the evolution of the world income distribution, Journal of Economic Perspectives, 19-36.

Kögel, T., and A. Prskawetz, 2001, Agricultural productivity growth and escape from the Malthusian trap, Journal of Economic Growth 6, 337-357.

Kraay, A., and C. Raddatz, 2007, Poverty traps, aid, and growth, Journal of Development Economics 82, 315-347.

Kremer, M., A. Onatski, and J. Stock, 2001, Searching for prosperity, Carnagie-Rochester Conference Series on Public Policy 55, 275-303.

Lee, R., 2003, The Demographic Transition: Three Centuries of Fundamental Change, Journal of Economic Perspectives 17, 167-190.

Lucas, R.E. Jr., 2000, Some macroeconomics for the 21st century, Journal of Economic Perspectives 11(4), 159-168

Malthus, T.R., 1798, An Essay on the Principle of Population.

Nelson, R., 1956, A theory of the low-level equilibrium trap in under-developed economies, American Economic Review 46, 894-908.

Pomeranz, K., 2000, The Great Divergence: China, Europe, and the Making of the Modern World Economy, Princeton University Press, Princeton.

Pritchett, L., 1997, Divergence, big time, Journal of Economic Perspectives 
Quah, D.T., 1993, Galton's fallacy and tests of the convergence hypothesis, Scandinavian Journal of Economics 95, 427-443.

Quah, D.T., 1996, Empirics for economic growth and convergence, European Economic Review 40, 1353-1375.

Reher, D.S., 2004, The Demographic transition revisited as a global process, Population, Space and Place 10, 19-41.

Silverman, B., 1981, Using kernel density estimates to investigate multimodality, Journal of the Royal Statistical Society Series B 43, 97-99.

Strulik, H., 2004, Economic growth and stagnation with endogenous health and fertility, Journal of Population Economics 17, 433-453.

Strulik, H., 2008a, Geography, health, and the pace of demo-economic develoment, Journal of Development Economics 86, 61-75.

Strulik, H., 2008b, Degrees of develoment, Discussion Paper, University of Hannover.

Strulik, H. and Weisdorf, J., 2008, Population, Food, and Knowledge: A Simple Unified Growth Theory, Journal of Economic Growth 13, 169-194.

United Nations, Department of Economic and Social Affairs, Population Division, World Population Prospects: The 2008 Revision, New York, 2009, http://data.un.org/.

Vollmer, S., H. Holzmann, F. Ketterer and S. Klasen, 2009, Diverging Convergence in Unified Germany, Discussion Paper, University of Hannover.

Wilson, C., 2001, On the scale of global demographic convergence 1950-2000, Population and Development Review 27, 155-171. 
Figure 3: High-Fertility Regimes and Low-Fertility Regimes Around the World: 1950- 1955 Vs. 2000-2005
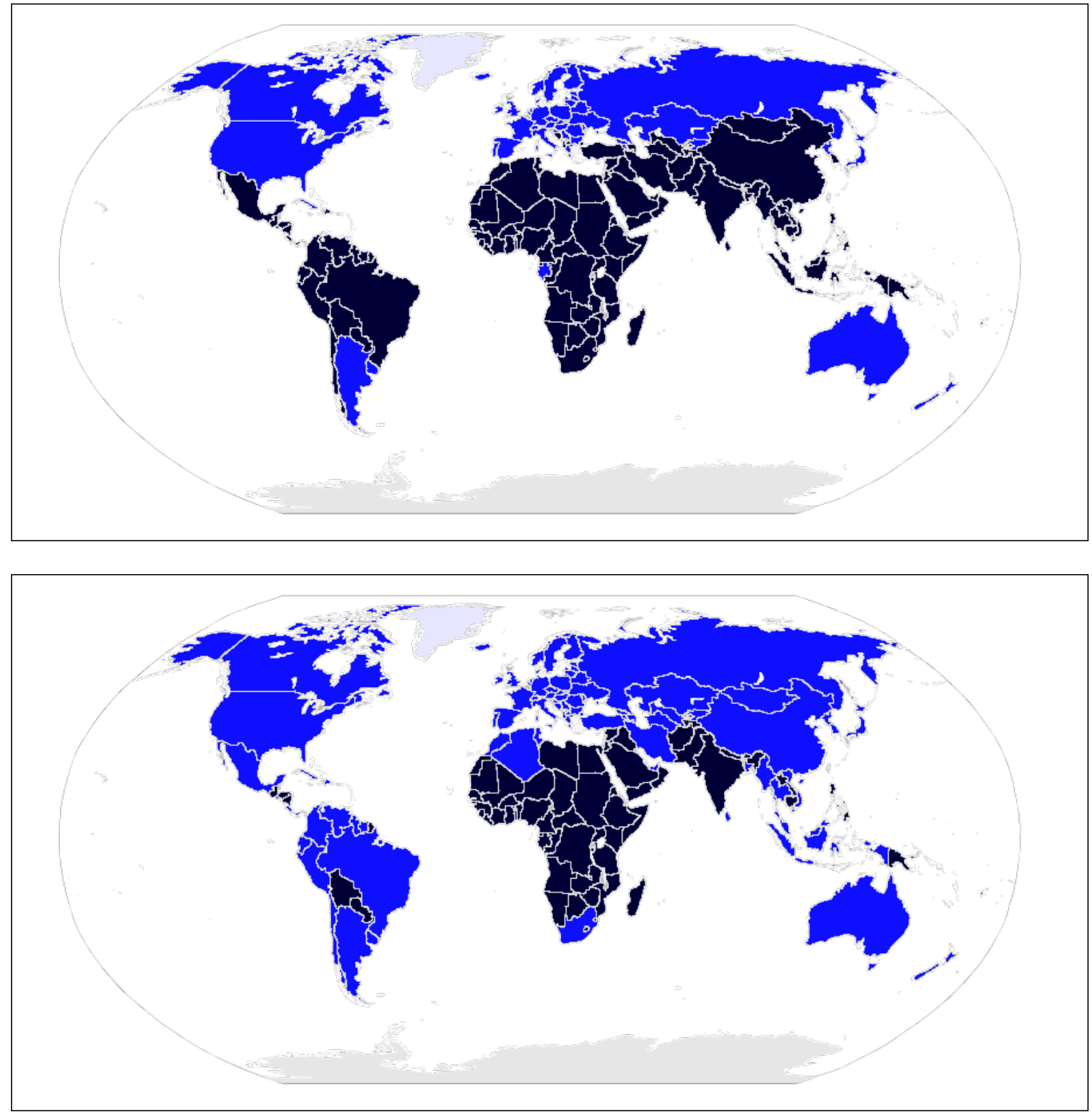

Note: The upper map shows the assignments of countries to the two fertility regimes in 1950-1955, the lower map shows the assignments in 2000-2005. Light blue color represents the low fertility regime, whereas the dark blue color represents the high fertility regime. 
Figure 4: Cross-Country Distribution of Fertility Rates:

1955-1955 - 1995-2000
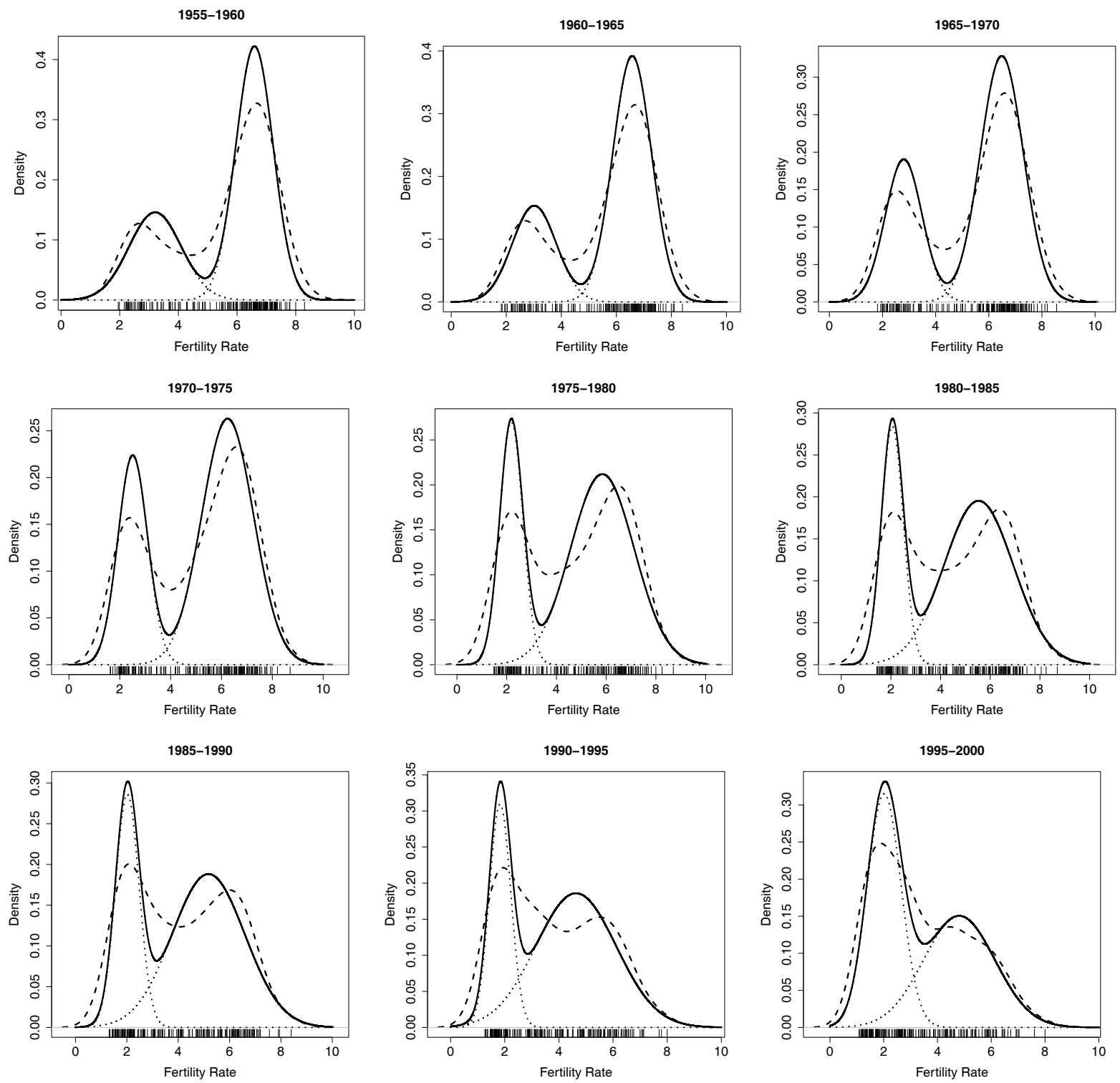

Note: Fitted mixture model (solid line), weighted components (dotted lines), and kernel density estimator (dashed line). The rugs below the plots represent the observed fertility rates. 